\title{
RELAÇÃO ENTRE NÍVEL DE ATIVIDADE FÍSICA E REALIZAÇÃO DE ATIVIDADES INSTRUMENTAIS EM FREQUENTADORES DE CENTRO DIA GERIÁTRICO
}

\author{
Jessica Rodrigues Pereira, Universidade Estadual Paulista - UNESP, Rio Claro - Brasil \\ Camila Vieira Ligo Teixeira, Universidade Estadual Paulista - UNESP, Rio Claro - \\ Brasil \\ Danilla Icassatti Conazza, Universidade Estadual Paulista - UNESP, Rio Claro - Brasil \\ Thays Martins Vital, Universidade Estadual Paulista - UNESP, Rio Claro - Brasil \\ Salma Stéphany Soleman Hernandez, Universidade Estadual Paulista - UNESP, Rio \\ Claro-Brasil \\ Florindo Stella, Universidade Estadual Paulista - UNESP, Rio Claro - Brasil
}

\section{RESUMO}

Objetivo: Analisar a associação entre nível de atividade física e a realização de atividades instrumentais de idosos frequentadores de Centro-Dia Geriátrico. Métodos: Estudo de delineamento transversal, com amostra constituída por 31 idosos frequentadores de Centro-Dia Geriátrico. Foram utilizados o Questionário de Baeck Modificado para Idosos (QBMI) e o Questionário de Atividades Instrumentais de Pfeffer (Pfeffer), para avaliar o nível de atividade física e realização de atividades instrumentais, respectivamente. E para caracterização da amostra, foi utilizado o Mini Exame do Estado Mental. Para análise dos dados foi utilizada a estatística descritiva e Correlação de Spearman. Resultados: A análise mostrou correlação moderada e negativa entre o QBMI e o Pfeffer $(\mathrm{r}=-0,04)$, podendo essa relação ser extrapolada para quanto maior o nível de atividade física, maior a independência na realização de atividades instrumentais. Conclusões: O nível de atividade física tem relação com a realização de atividades instrumentais. Assim, o incentivo à prática de atividade física é de extrema importância para a população idosa, para que a mesma se mantenha independente.

Palavras-Chave: Centro-dia geriátrico; Atividades instrumentais; Nível de atividade física; Funções executivas.

\section{RELATIONSHIP BETWEEN PHYSICAL ACTIVITY AND PERFORMANCE OF INSTRUMENTAL ACTIVITIES IN PATRONS OF GERIATRIC DAYCARE CENTERS}

\begin{abstract}
Objective: Analyze the association between physical activity level and performance of instrumental activities of elderly from a Geriatric Day-Care. Methods: It is a crosssectional design study, with a sample of 31 elderly from a Geriatric Day-Care. It was used the Modified Baeck questionnaire for Elderly (QBMI)and the Instrumental Activities Questionnaire Pfeffer (Pfeffer) to evaluate physical activity level and instrumental activities performance, respectively. And to characterize the sample, it was used the Mini
\end{abstract}


Mental State Examination. For data analysis, it was used descriptive statistics and Spearman correlation. Results: The analysis showed a negative correlation between moderate and QBMI and Pfeffer $(\mathrm{r}=-0.04)$, which can be extrapolate to the higher level of physical activity, the greater independence in performing instrumental activities. Conclusions: The level of physical activity is related to the performance in instrumental activities. Thus, encouraging physical activity is extremely important for the elderly population, so that it remains independent.

Key-Words: Geriatric day-care, Instrumental activities, Physical Activities level, Executive functions. 


\section{INTRODUÇÃO}

Tanto no Brasil, como nos demais países em desenvolvimento, há um aumento significativo da expectativa de vida, repercutindo nos campos sociais e econômicos do país. O envelhecimento com menor independência representa um problema social para os sistemas previdenciários e de saúde. ${ }^{1}$

O envelhecimento normal engloba um declínio gradual nas funções cognitivas, dependentes de processos neurológicos que se alteram com a idade. ${ }^{2}$

Funções cognitivas podem ser definidas como as fases do processo de informação, como percepção, aprendizagem, memória, atenção, vigilância, raciocínio e solução de problema, além disso, incluiu o funcionamento psicomotor (tempo de reação, tempo de movimento, velocidade de desempenho) neste conceito. ${ }^{3}$

No envelhecimento normal ocorrem discretas alterações nas funções cognitivas, que não causam prejuízos importantes nas atividades de vida diária. Segundo as mesmas autoras, o conhecimento adquirido ao longo da vida continua preservado até por volta dos 70 anos, a partir de então pode ocorrer redução importante nas habilidades praticas e executivas, que dependem da percepção visual, análise visuoespacial e desempenho visuomotor. Estas reduções são consequências de mudanças na atenção memória imediata e na capacidade de planejamento antecipatório de ações. Ainda a literatura mostra que os processos baseados em habilidades cristalizadas, como conhecimento verbal e compreensão continuam mantidos ou melhoram com o envelhecimento. Em contrapartida, processos baseados em habilidades fluídas, tais como tarefas aprendidas, mas não executadas, sofrem declínios mais constantes. ${ }^{1,4-6}$

Existem fatores de risco que podem aumentar a predisposição de um indivíduo ao prejuízo cognitivo. Dentre estes fatores estão nível educacional e sedentarismo, destacando-se também idade, gênero, histórico familiar, tabagismo, etilismo, estresse, aspectos nutricionais, socialização, entre outros. ${ }^{7}$ 
A população idosa está inserida no segmento que tradicionalmente não se engaja em práticas de exercícios físicos, entretanto, estudos têm demonstrado que o exercício físico pode diminuir o risco de declínio cognitivo e ainda melhorar suas funções executivas em associação a benefícios fisiológicos. ${ }^{1,7-10}$ Este é um fator de estrema importância para a manutenção da plasticidade e saúde do cérebro, pois promove constante desafio intelectual. $^{10}$

O risco de desenvolvimento de declínio cognitivo é diretamente proporcional à idade e inversamente proporcional ao nível educacional. ${ }^{11}$ Portanto, mesmo que o idoso tenha baixa escolaridade, é importante estimular suas funções cognitivas, preservando-as, seja através de atividades físicas ou cognitivas, pois nessa população os declínios tendem a ser mais severos e recorrentes. ${ }^{12}$

O nível de atividade física pode ser relacionado às atividades instrumentais, pois ambos encontram-se entre as áreas que compõe a funcionalidade, sendo que ambos são necessários para realização das Atividades Básicas da Vida Diária. ${ }^{13}$

Na tentativa de auxiliar a população idosa, métodos de atenção à saúde foram criados no sentido de proteção e inclusão social. Dentre os serviços de proteção social, inclui-se a modalidade Centro-Dia Geriátrico. Esta modalidade oferece atendimento multiprofissional aos idosos, desenvolvendo atividades relacionadas à promoção e proteção de saúde, incentivando também a socialização dos frequentadores. O Centro-Dia Geriátrico é destinado à permanência diurna de idosos com dependência parcial nas atividades de vida diária e que necessitam de assistência multiprofissional. ${ }^{14}$

O que leva às famílias à procurar um centro dia geriátrico é fato do mesmo ser um local único, capaz de proporcionar consultas com especialistas e intervenções para indivíduos com múltiplas necessidades, que teriam que recorrer a diversas modalidades de assistência à saúde para sanar suas demandas. ${ }^{15}$ 
Desta forma, o objetivo deste trabalho foi analisar a associação entre nível de atividade física e a realização de atividades instrumentais de idosos frequentadores de Centro-Dia Geriátrico.

\section{MÉTODOS}

\section{Delineamento da Pesquisa}

O presente estudo teve caráter descritivo, com corte transversal.

\section{Procedimentos}

O presente projeto de pesquisa foi encaminhado ao Comitê de Ética em Pesquisa do Instituto de Biociências, UNESP, Campus de Rio Claro, São Paulo, sob Protocolo n5020 juntamente com o Termo de Consentimento Livre e Esclarecido,conforme normas do Conselho Nacional da Saúde (resolução 196/96), com coleta de assinatura no mesmo, para aqueles que aceitaram participar do estudo.

\section{Amostra}

Participaram do estudo 31 idosos ( 8 homens e 24 mulheres) freqüentadores do Centro Dia do Idoso "Padre Casagrande", Rio Claro, SP.

\section{Protocolo de Avaliação}

Mini-Exame do Estado Mental (MEEM)- Utilizado a fim de rastrear o estado cognitivo global, avaliando funções como orientação, memória imediata, atenção e cálculo, evocação e linguagem. Como o MEEM sofre influência da escolaridade, valores de referência foram propostos com o objetivo de distinguir sujeitos com possíveis déficits cognitivos. A análise de uma amostra brasileira sugeriu os seguintes valores para estudos em nosso meio: para analfabetos $=20$ pontos; de 1 a 4 anos de escolaridade $=25$; de 5 a 8 anos=26,5; de 9 a 11 anos=28; e para indivíduos com escolaridade superior a 11 anos=29 pontos. ${ }^{16-17}$

Questionário de Baecke Modificado Para Idosos (QBMI) - Abrange três áreas básicas: atividades domésticas, atividades esportivas e atividades de lazer, a fim de estimar o nível de atividade física dos participantes. ${ }^{18}$ 
Questionário de Atividades Instrumentais de Pfeffer (Pfeffer) - Evidencia a funcionalidade através do grau de independência para realização das atividades instrumentais de vida diária. É constituído por itens relacionados à capacidade do individuo de realizar atividades da vida diária e funções cognitivas. ${ }^{20}$

\section{Análise estatística}

Foram realizadas análises descritivas dos dados (média e desvio-padrão), bem como, visto que o QBMI não apresenta dados de natureza escalar, foi realizada a verificação da distribuição dos dados por meio do teste Shapiro Wilk, que rejeitou a hipótese de normalidade.

Devido à natureza escalar dos demais dados, foi utilizada a estatística não paramétrica Correlação de Spearman. A Análise foi realizada em ambiente estatístico SPSS (Statistical Package for Social Sciences) 15.0. O nível de significância adotado foi de $\mathrm{p}<0,05$. O teste Kruskall Walis foi utilizado para comparar MEEM dos grupos dentre os diferentes níveis de escolaridade.

\section{RESULTADOS}

Os resultados obtidos pelos participantes foram divididos de acordo com a proposta ${ }^{1}$ para o MEEM, a fim de neutralizar as diferenças que o nível de escolaridade poderia trazer na cognição global, e são apresentados na Tabela 1.

Tabela 1 - Médias de idade e escolaridade e MEEM dos grupos divididos por escolaridade $^{17}$

\begin{tabular}{cccc}
\hline Divisão escolaridade & $\begin{array}{c}\text { Escolaridade } \\
\text { Média }\end{array}$ & Idade & MEEM \\
\hline Analfabetos $(\mathrm{n}=6)$ & 0 & $78,50 \pm 8,12$ & $20,42 \pm 6,26$ \\
$1-4$ anos $(\mathrm{n}=24)$ & $2,75 \pm 1,15$ & $77,21 \pm 9,48$ & $22,25 \pm 4,62$ \\
$5-8$ anos $(\mathrm{n}=3)$ & $5,67 \pm 0,58$ & $73,33 \pm 6,66$ & $22,50 \pm 3,50$ \\
$9-11$ anos $(\mathrm{n}=3)$ & $9,67 \pm 0,58$ & $72 \pm 12,12$ & $26,67 \pm 2,31$ \\
\hline
\end{tabular}


No entanto, o teste Kruskall Walis não mostrou diferença estatisticamentesignificativa na média das variáveis idade e escore do MEEM entre os grupos. Assim, todos os sujeitos fora agrupados em um único grupo para a correlação.

O teste de correlação de Spearman mostrou relação negativa e moderada $(r=-0,4)$ entre o QBMI e o Pfeffer. Mostrando que quanto maior a pontuação no QBMI, menor a pontuação no Pfeffer. Sabendo que maior pontuação no QBMI e Pfeffer significa maior nível de atividade física e maior dependência para a realização de atividades instrumentais, respectivamente. Assim podemos extrapolar essa relação para quanto maior o nível de atividade física, maior a independência na realização de atividades instrumentais.

\section{DISCUSSÃO}

Analisando os valores médios do QBMI, embora não haja pontos de corte para tal questionário, podemos observar que o nível de atividade física apresentado pelos participantes do presente estudo é baixo, quando comparados a outros estudos sobre idosos da comunidade. ${ }^{20}$ Esses resultados corroboram os dados da literatura quando relacionados a idosos institucionalizados, ${ }^{21}$ cabendo aqui a ressalva que de que a instituição Centro-Dia do Idoso não é uma instituição de longa permanência.

A relação aqui apresentada entre QBMI e Pfeffer foi negativa e moderada. Essa relação parece mostrar que o nível de atividade física pode interferir na realização de atividades instrumentais.

As atividades instrumentais necessitam de planejamento, sequência, organização, ajustes, abstração e motivação entre outros. ${ }^{22} \mathrm{O}$ mesmo estudo avaliou arranjos domiciliares, e demonstrou que idosos realmente tendem a perder a capacidade de realização de atividades instrumentais com o passar dos anos, assim, manter o nível cognitivo, ajudaria a mantê-las.

Em nosso estudo, o nível de atividade física parece ter associação com as atividades instrumentais, o que nos leva acreditar que idosos fisicamente ativos mantém sua capacidade de realizar atividades instrumentais preservada, e que o baixo nível de 
atividade física, associado à baixa cognição global prejudicam a realização das atividades instrumentais. $^{22}$

Quando os idosos apresentam declínio cognitivo, a primeira habilidade perdida é a de realizar tarefas complexas (atividades instrumentais) e depois as atividades da vida diária. Os autores destacam ainda, que esses idosos poderão, posteriormente, ser acometidos pela demência. $^{23}$

O exercício interfere no desempenho cognitivo, pois parece gerar um aumento de neurotransmissores devido à mudanças nas estruturas cerebrais, o que pode causar melhora cognitiva em indivíduos com prejuízo mental. ${ }^{1}$ Entretanto, em idosos com a cognição considerada normal, essa melhora é limitada. Embora diversos trabalhos ${ }^{24}$ demonstrem que a prática de exercícios pode levar á melhora da cognição, mesmo em idosos sem declínio.

O envolvimento numa prática regular de atividade física, não exerce por si só um efeito protetor sobre a cognição, mas pode ser visto como um marcador de boa saúde, relacionado com o menor risco de declínio cognitivo e demência. ${ }^{10,25}$

Uma vida fisicamente ativa, não está somente relacionada à atividade física sistematizada, mas também a atividades de lazer, e praticá-las é benéfico não só para o aumento do nível de atividade física, como também para o estado cognitivo global. ${ }^{26}$

\section{CONCLUSÃO}

Diante do exposto parece que o nível de atividade física tem relação com a realização de atividades instrumentais. $\mathrm{O}$ que nos leva a acreditar que o incentivo à prática de atividade física é de extrema importância para a população idosa, para que a mesma se mantenha independente.

\section{REFERÊNCIAS}

${ }^{1}$ ANTUNES, H. K. M et al. Alterações cognitivas em idosas decorrentes do exercício físico sistematizado. Revista da SOBAMA, São Paulo, v. 6, n, 1, p. 27-33, dez. 2005. 
${ }^{2}$ CANINEU, P. R. et al. Terapia multidisciplinar: uma proposta de tratamento global do idoso. Mundo Saúde, v. 29, n. 4, p. 662-665, 2005.

${ }^{3}$ CHODZKO-ZAIJKO, W. J.; MOORE, K. A. Physical fitness and cognitive functioning in aging. Exercise and Sport Sciences Reviews, Hagerstown, v. 22, p. 195-220, 1994.

${ }^{4}$ CAMARGO, C. H. P.; GIL; G., MORENO, M. D. P. Envelhecimento "normal" e cognição. In: BOTTINO, C. M. C.; LAKS, J.; BLAY, S. L. Demência e transtornos cognitivos em idosos. Rio de Janeiro: Guanabara Koogan, 2006.

${ }^{5}$ PARENTE, M. A.; WAGNER G. Teorias abrangentes sobre envelhecimento cognitivo. In: PARENTE, M. et al. Cognição e envelhecimento. Porto Alegre: Artmed, 2006. p. 3145.

${ }^{6}$ ARGIMON, I. I. L. et al. Funções executivas e a avaliação de flexibilidade de pensamento em idosos. Revista Brasileira de Ciências do Envelhecimento Humano, Passo Fundo, p. 35-42, jul./dez. 2006.

${ }^{7}$ ANTUNES, H. K. M. et al. Exercício físico e função cognitiva: uma revisão. Revista Brasileira de Medicina do Esporte, São Paulo, v. 12, n. 2, 2006.

${ }^{8}$ COLCOMBE, S.; KRAMER, A. F. Fitness effects on the cognitive function of older adults: a meta-analytic study. Psychological Science, v.14, 125-130, 2003.

${ }^{9}$ CASSILHAS, R. et al. The impact of resistance exercise on the cognitive function of the elderly. Medicine and Science in Sports and Exercise, v. 39, n. 8, p. 1401-1407, 2007.

${ }^{10}$ LAURIN, D. et al. Physical activity and risk of cognitive impairment and dementia in elderly persons. Archives of Neurology, Chicago, v. 58, p. 498-504, 2001. 
${ }^{11}$ GONÇALVES, M. P.; TOMAZ, C.; SANGOI, C. Considerações sobre envelhecimento, memória e atividade física. Revista Brasileira de Ciência e Movimento, Brasília, v. 14, n. 1, p. 101-108, 2006.

${ }^{12}$ YLIKOSKI, R. et al. Heterogeneity of cognitive profiles in aging: successful aging, normal aging, and individuals at risk for cognitive decline. European Journal of Neurology, Oxford, v. 6, n. 6, p. 645-652, 1999.

${ }^{13}$ AGÜERO-TORRES, H. et al. Dementia is the major cause of functional dependence in the elderly: 3-year follow-up data from a population-based study. American Journal of Public Health, Boston, v. 88, n. 10, p.1452-1456, 1998.

${ }^{14}$ BRASIL. Ministério da Saúde. Portaria do Gabinete do Ministro de Estado da Saúde de n. 1395, de 9 dez. 1999, que aprova a Política Nacional de Saúde do Idoso e dá outras providências. Diário Oficial da República Federativa do Brasil, 13 dez, 1999.

${ }^{15}$ FRANCIULLI, S. E. et al. A modalidade de assistência centro-dia geriátrico: efeitos funcionais em seis meses de acompanhamento multiprofissional. Ciência \& Saúde Coletiva, Rio de Janeiro, v. 12, n. 2, p. 373-380, 2007.

${ }^{16}$ FOLSTEIN, M.; FOLSTEIN, S.; MCHUGH, P. Mini-mental state: a practical method for grading the cognitive state off patients for the clinician. Journal of Psychiatry Research, v.12, p. 189-98, 1975.

${ }^{17}$ BRUCKI, S. M. D. et al. Sugestões para o uso do mini-exame do estado mental no Brasil. Arquivos de Neuro-Psiquiatria, v. 61, n. 3, p. 777-781, 2003.

${ }^{18}$ VOORRIPS, L. et al. A physical activity questionnaire for elderly. Medicine and Science in Sports and Exercise, Hagerstown, v. 23, n. 8, p. 974-979, 1991. 
${ }^{19}$ PFEFFER, R. I.; KUROSAKI, T. T.; HARRAH, C. H. L. Measurement of functional activities in older adults in the community. Journal of Gerontology, Washington, v. 37, p. 323-329, 1982.

${ }^{20}$ SEGHERS, J. et al. Habitual level of physical activity and muscle fatigue of the elbow flexor muscles in older men. European Journal of Applied Physiology, Heidelberg, v. 89, p. 427-434, 2003.

${ }^{21}$ QUADROS-JUNIOR, A. C. et al. Estudo do nível de atividade física, independência funcional e estado cognitivo de idosos institucionalizados: análise por gênero. Brazilian Journal of Biomotricity, n. 2, p. 39-50, 2008.

${ }^{22}$ OLIVEIRA, S. F. D. Declínio cognitivo, funcionalidade, e arranjos domiciliares entre os idosos do Município de São Paulo. 2006. Dissertação (Mestrado em Enfermagem) - Escola de Enfermagem, Universidade de São Paulo, São Paulo, 2006.

${ }^{23}$ MARRA, T. A. et al. Avaliação das atividades de vida diária de idosos com diferentes níveis de demência. Revista Brasileira de Fisioterapia, São Carlos, v. 11, n. 4, p. 267$73,2007$.

${ }^{24}$ HEYN, P.; ABREU, B. C.; OTTENBACHER, K. J. The effects of exercise training on elderly persons with cognitive impairment and dementia: a meta-analysis. Archives Physical Medicine and Rehabilitation, v. 85, p. 1694-704, 2004.

${ }^{25}$ WEUVE J, et al. Cumulative exposure to lead in relation to cognitive function in older women. Environmental Health Perspectives, Research Triangle Park, v. 117, p. 574580, 2009.

${ }^{26}$ VERGHESE $\mathrm{J}$, et al. Leisure activities and the risk of amnestic mild cognitive impairment in the elderly. Neurology, Minneapolis, v. 66, n. 6, p. 821-827, mar. 2006.

Conexões: revista da Faculdade de Educação Física da UNICAMP, Campinas, v. 10, n. 3, p. 20-31, set./dez. 2012.30 ISSN: 1983-9030 


\section{AGRADECIMENTOS}

Laboratório de Atividade Física e Envelhecimento - LAFE

Conselho Nacional de Desenvolvimento Científico e Tecnológico - CNPq 